\title{
Configuración teórica de la noción de Discurso Digital Escolar relativo a la Química
}

\section{The theoretical makeup / configuration of Scholastic Digital Discourse in Chemistry}

Néstor Alexander Zambrano-González

'Universidade Estadual de Campinas (UNICAMP), Programa de Pós-Graduação em Educação, Campinas, SP, Brasil. E-mail: n229930@dac.unicamp.br

Resumo: Este artículo tiene como objetivo presentar la configuración teórica de la noción de Discurso Digital Escolar relativo a la Química (DDErQ, por sus siglas en español), propuesta que, tomando como ángulo de entrada la formación inicial de profesores en el campo de la Educación Química, busca constituirse lugar posible para la interpretación, la comprensión y la resignificación de las futuras prácticas escolares asociadas a la enseñanza de esta ciencia. En este propósito, los principales referenciales teóricos que configuran esta noción se derivan, por una parte, del Análisis del Discurso en su vertiente iniciada en Francia por Pêcheux y desarrollada ampliamente en Brasil por Eni Orlandi y, por la otra, de los desdoblamientos que, basados en esta filiación teórica, fueron publicados en el campo de las llamadas ciencias de la naturaleza por Almeida y en el campo de las nuevas tecnologías del lenguaje por Dias.

Palavras-chave: Enseñanza de la química; Discurso digital; DDErQ; Formación inicial de profesores; Memoria digital.

Abstract: This paper aims to present the theoretical configuration of the notion of Scholastic Digital Discourse related to Chemistry (DDErQ, by its acronym in Spanish), a proposal that seeks to constitute a possible place for the interpretation, understanding and resignification of future school practices associated with the teaching of this science by taking as an entry point/perspective the Initial Teacher Training in the field of Chemical Education. According to this proposal, the main theoretical references that make up this notion are derived, on the one hand, from the Discourse Analysis in its aspect initiated in France by Pêcheux and developed widely in Brazil by Eni Orlandi. And, on the other hand, derive from branches that, based on this theoretical affiliation, were published in the field of so-called natural sciences by Almeida, and in the field of new language technologies by Dias.

Keywords: Chemistry teaching; Digital discourse; Initial teacher training; Digital memory.

Recebido em: 23/10/2020

Aprovado em: 31/05/2021 


\section{Preámbulo}

"La comunicación en un sentido amplio fue resignificada por lo digital y por sus dispositivos (smartphones, e-readers, aplicaciones, redes sociales, etc.). Esa resignificación se vincula al lenguaje, la escritura, la lectura y al texto" (DIAS, 2019, p. 55), y podría afirmarse que, sin lugar a duda, sus efectos han permeado diferentes escenarios en que son desarrolladas las actividades de la vida humana, uno de ellos, el científico-escolar.

En esta perspectiva, pensar la escuela como un "[...] lugar de elaboración de conocimiento y de su colocación a la disposición de los diferentes individuos que son parte de la sociedad" (ORLANDI, 2008b, p. 159, traducción nuestra), pero sobre todo como un espacio significante en el que a través de la repetición y de la regularización se pretende dar cierta 'estabilidad' al sentido, implica entre otros aspectos, reconocer al profesor como un sujeto (un ser simbólico), inscrito en la historia, exento de neutralidad, que forma parte de una institución consolidada socio-históricamente por la cual circula una versión polisémica del conocimiento como objeto de enseñanza producto, entre otras posibilidades, del efecto metafórico (PÊCHEUX, 1969) ${ }^{1}$, y que para comunicarse utiliza el lenguaje que cabe destacar, no es transparente ni libre de equívocos.

Este artículo tiene como objetivo presentar la configuración teórica de la noción de Discurso Digital Escolar relativo a la Química (DDErQ, por sus siglas en español), propuesta que, tomando como ángulo de entrada la Formación Inicial de Profesores en el campo de la Educación Química, busca constituirse lugar posible para la interpretación, la comprensión y la resignificación de las futuras prácticas escolares asociadas a la enseñanza de esta ciencia. En este propósito, se parte del reconocimiento de la Química como una ciencia históricamente constituida y provista de una estructura conceptual especializada que le permite indagar sobre variados fenómenos de la materia en los niveles macroscópico y submicroscópico y representarlos en el nivel simbólico, y de la transversalización del carácter humanístico discursivo en los procesos de enseñanza y de aprendizaje de los futuros profesores, en el contexto de su formación inicial. Posteriormente, se exponen los pilares de orden conceptual que configuran el DDErQ estrechamente vinculado a las nociones de Discurso Escolar relativo a la Ciencia y de Discurso Digital tejidas en la filiación teórica de la vertiente pechêuxtiana de Análisis del Discurso y, finalmente, se plantean las primeras aproximaciones acerca de sus características, de sus modos de producción y de sus posibles efectos en la enseñanza escolar de esta ciencia.

\section{Historicidad de la noción de DDERQ: un lugar para la relación entre química y formación inicial de profesores de química}

La Química surge como ciencia en el siglo XVII, pero su historia data de tiempos antes de Cristo. En su definición escolar más amplia, básica y circulada se presenta como "[...] la ciencia que se ocupa del estudio de la materia y de los cambios que acontecen en ella" (CHANG; GOLDSBY, 2013, p. 2). En la actualidad, es considerada como una ciencia que desde la educación secundaria y media contribuye a la adquisición de conocimientos científicos básicos para el ejercicio de la ciudadanía y que, en algunos 
programas de educación superior no especializados en el área, proporciona fundamentos teóricos y prácticos indispensables para el ejercicio de determinadas actividades de orden profesional.

En cuanto a su enseñanza en los diferentes niveles del sistema educativo cabe añadir que, mayoritariamente, ésta se halla bajo la responsabilidad de profesionales formados en las instituciones de educación superior y que sus currículos se encuentran organizados siguiendo, entre otras, propuestas en torno a su estructura conceptual como la del célebre Triplete de la Química, de Johnstone (1982), compuesto por los niveles macroscópico, submicroscópico y representacional / simbólico; formulación que en la agenda de la filosofía de la Química ha sido estudiada (TALANQUER, 2011), criticada (LABARCA, 2009), ampliada (SJÖSTRÖM, 2013) e incluso reformulada (TABER, 2013), entre otras razones, dadas sus escasas contribuciones a la historización de los conocimientos químicos los cuales para ser comprendidos deben fundamentarse en elementos filosóficos más amplios que los propios conceptos científicos.

En este encuadramiento conceptual que corresponde con una perspectiva holística circunscrita en las tendencias contemporáneas sobre la formación del profesorado de ciencias (CARVALHO; GIL-PÉREZ, 2011), se propone tomar como ángulo de entrada de la noción de Discurso Digital Escolar relativo a la Química al campo de estudio de la Educación Química, el contexto de la formación inicial de los profesores del área, en el nivel universitario, considerándolo como un escenario de preparación interdisciplinar fértil para la apropiación de fundamentos científicos, filosóficos, humanísticos, pedagógicos y didácticos; para incentivar la reflexión y la transformación y para desarrollar las competencias necesarias para desempeñar asertivamente su futuro papel en la enseñanza escolar de esta ciencia.

Refiriéndose a la formación docente, en 2004, el profesor Vicente Talanquer publicó un artículo en el que discutía ¿Qué conocimiento distingue a los buenos maestros de química? En él planteó los conocimientos y habilidades que, en su perspectiva, caracterizan al docente de Química en contraste con docentes de otras disciplinas y con otros profesionales de la Química. En sus palabras:

La respuesta es una amalgama entre conocimiento disciplinario, didáctica y pedagogía. Es el resultado de 'pensar en química' con el propósito de motivar, sorprender, despertar la curiosidad, generar interés y dar sentido; es la consecuencia de la reflexión constante sobre la naturaleza de los temas, ejemplos, explicaciones, analogías, metáforas, representaciones, actividades, experiencias, preguntas, problemas, que son apropiadas para diversos tipos de estudiantes y pueden favorecer aprendizajes más significativos. (TALAQUER, 2004, p. 61).

En esa idea de 'pensar en química' con el propósito de 'dar sentido'2 (ORLANDI, 1996, p. 28), la noción de DDErQ busca contribuir en la configuración de un escenario formativo del profesorado en el que lo digital representa un espacio de transformación de las condiciones de producción que incide en las estructuras de la formalización de los saberes y en las relaciones sociohistóricas establecidas en el marco de los dinámicos procesos de enseñanza y de aprendizaje.

${ }^{2}$ Cabe aclarar que con esta acción no se considera que el sentido sea impuesto, único, estático ni unívoco, toda vez que, de acuerdo con la autora, "no hay relación término-a-término entre las cosas y el lenguaje". 


\section{Un punto de partida: los orígenes de la noción de Discurso Digital Escolar relativo a la Química}

La génesis de la noción de Discurso Digital Escolar relativo a la Química ocurre en el campo de las ciencias de la naturaleza con Almeida (2010, p. 21, traducción nuestra) quien introdujo la noción de "Discurso Escolar relativo a la Ciencia" que, de acuerdo con la autora, es una interface posible entre el discurso científico y el discurso escolar que implica, en sus palabras, reconocer la existencia de un discurso propio de la ciencia (el discurso científico), con posibilidad de múltiples interpretaciones, asociadas a las memorias discursivas de los individuos que lo interpretan. Subjetivo que debe mantener muchas interfaces con el discurso científico propiamente dicho, y destinado a, por un lado, facilitar la interlocución con quien busca interpretarlo, y por otro, satisfacer la propuesta de posibilitar una comprensión de la ciencia como construcción humana. (ALMEIDA, 2010).

En su texto de formulación de la noción de Discurso Escolar relativo a la Ciencia, Almeida (2010, p. 21, traducción nuestra) anotó: "[...] Pero una restricción al propio título de este ítem aún es necesaria: la de que, en verdad, no se puede hablar genéricamente de la ciencia; siendo que cada ciencia particular posee sus características propias y sus modos de producción".

Esta anotación constituyó la base para tejer un primer desdoblamiento que conduce a la noción intermediaria de Discurso Escolar relativo a la Química, sin que su localización en este campo de conocimiento implique desconocer las características atribuidas por la autora a la noción base de Discurso Escolar relativo a la Ciencia que, como cabe esperar, junto con los principios teórico-analíticos de la vertiente pechêuxtiana de Análisis del Discurso (AD), configurarán el punto de partida en la tarea de comprender las características propias y los modos de producción del DDErQ.

En este punto conviene agregar que la noción de DDErQ avanza en relación con la noción propuesta por Almeida (2010) en el abordaje de las especificidades que dentro de la enseñanza de las ciencias tiene la enseñanza de la Química, y en la localización como objeto de análisis en las prácticas formativas iniciales del profesorado, de lo digital. Y pensado a partir de su constitución, su formulación y su circulación tal como lo planteó al describir los procesos de producción del discurso Orlandi (2008b) y posteriormente lo retomó Dias (2018) en la estructuración de su desdoblamiento teórico respecto al Análisis del Discurso Digital.

Antes de continuar, es pertinente aclarar que el uso de la connotación 'escolar' no quiere decir que la noción de DDErQ quede restringida a las prácticas que acontecen en la enseñanza fundamental o media, ni tampoco que se pretenda considerar a los sujetos (profesor, estudiante) aislados de sus condiciones de producción en los procesos discursivos. Por el contrario, el propósito del uso de la adjetivación 'escolar' en este contexto deberá ser entendido en función del proceso de formación institucionalizado (inscrito socio-históricamente en una esfera de pensamiento dominante), que acontece durante diferentes etapas de la vida a través de los distintos niveles de enseñanza en el que tanto profesores como estudiantes producen colectivamente en los términos pechêuxtianos efectos de sentido entre locutores (ORLANDI, 2003), que se materializan en formaciones discursivas específicas, en este caso, de naturaleza científico-escolar. 
De la misma forma, cabe precisar que la inclusión de lo 'digital' no corresponde simplemente a la adición de un atributo en boga, pues al igual que Dias (2018, p. 28, traducción nuestra), en la noción de DDErQ, lo digital trasciende "[...] una mera forma de producción de la tecnología" para ser reconocido "[...] como una condición de producción [...] del discurso, como una condición y medio de producción y reproducción de las formas de existencia [...]", que tienen lugar en desarrollo de actividades escolares circunscritas en contextos globales de orden científico, político, ideológico, socioeconómico e institucional, por mencionar algunos, y, simultáneamente, en contextos delimitados vinculados a la práctica escolar y a la historia de vida de los sujetos que participan en dichos procesos formativos.

\section{Pilares teóricos de la noción de Discurso Digital Escolar relativo a la Química}

Con la noción de Discurso Escolar relativo a la Química derivada de la proposición de Discurso Escolar relativo a la Ciencia planteada por Almeida (2010), en la que tal como se mencionó, se conciben las especificidades de cada ciencia particular, trátese, por ejemplo, de la Biología, de la Química o de la Física, y se reconocen unos modos de producción que les son característicos. A continuación, se describen los cuatro pilares teóricos que configuran el DDErQ.

El primero emerge de considerar en la perspectiva discursiva a la escuela como espacio significante y al profesor en formación inicial como sujeto, lo que nos lleva a reflexionar sobre el papel del discurso en el campo de estudio de la Enseñanza de la Química.

De acuerdo con Orlandi (2008b, p. 14, traducción nuestra) "[...] el discurso es un proceso continuo que no se agota en una situación particular. Otras cosas fueron dichas antes y otras serán dichas después. Lo que tenemos son siempre 'pedazos', 'trayectos' estados del proceso discursivo", y es en esa abertura que procede cuestionar arraigadas ideas como la del discurso univoco, en otras palabras, aquel que solo tiene un significado, una interpretación, un único sentido posible, restringido a la literalidad, con obligación de ser objetivo y no ambiguo. O como la del discurso entendido simplemente como una producción coherente resultado del uso instrumental de una herramienta de comunicación para pensarlo desde otro lugar, por ejemplo, aquel que Orlandi (1996, p. 12, traducción nuestra) propone cuando señala que "[...] es por el discurso que mejor se comprende la relación entre lenguaje / pensamiento / mundo, porque el discurso es una de las instancias materiales (concretas) de esa relación".

Estableciendo como punto de partida esa relación lenguaje/pensamiento/ mundo, podría decirse que el discurso escolar proyecta un lugar de ciencia que no es cualquiera y que al adquirir forma material (textualización), representa una de las múltiples formulaciones discursivas posibles, en este caso, relativas a la Química.

En lo que respecta al proceso de enseñanza y de aprendizaje es clave destacar que en su desarrollo ocurren cambios en la formación discursiva que, entendida en los términos de Pêcheux (1988, p. 160, traducción nuestra) "[...] corresponde a un dominio de saber [en este caso, relativo a la Química], constituido de enunciados discursivos que representan un modo de relacionarse con la ideología vigente, regulando lo que puede y debe ser dicho", lo cual en la práctica escolar, significa que el sentido se constituye y se estabiliza en la relación entre los sujetos, es decir, el sentido no 
está predeterminado, sino que depende de la relación establecida entre profesores y estudiantes afectada por la lengua, la ideología y la tecnología, en función del propósito de la educación de estabilizar ciertos conocimientos mediante la repetición, el encuentro de interpretaciones, el juego de complejización, y el desplazamiento del decir. Movimientos que, entre otras, le confieren a determinado sujeto, la posibilidad dentro de otras posibilidades, de proyectar un lugar de 'comprensión' 3 de la ciencia (ORLANDI, 2006a).

Hecha esta colocación, el segundo elemento teórico está relacionado con aquello que sería el discurso digital, para lo cual vale recurrir a la definición de Orlandi (2009, p. 62-63, traducción nuestra):

\begin{abstract}
El lenguaje digital o el discurso electrónico, como prefiero llamar, reorganiza la vida intelectual, redistribuye los lugares de interpretación, desplaza el funcionamiento de la autoría y la propia concepción de texto. Pero no nos engañemos. Es aún una tecnología de la escritura. Tiene un impacto semejante al de la invención de la prensa. Pero difiere de esta por su naturaleza del punto de vista técnico, científico y administrativo, en términos sociales y políticos.
\end{abstract}

En este orden de ideas, se debe insistir en que en la construcción teórica de la noción de DDErQ lo 'digital' no debe ser entendido como un atributo asociado simplemente a un espacio material (internet) en el que circula información a la cual determinado 'usuario' tiene acceso, ni como un mero soporte para el proceso de enseñanza y de aprendizaje, o como un locus no significante, pues como afirma Dias (2018, p. 20, traducción nuestra), se apunta para la comprensión "[...] del discurso digital como objeto de análisis del análisis del discurso, una vez que lo digital se ha configurado como un campo de cuestiones imprescindible a las ciencias y al hacer científico".

Un tercer elemento teórico se deriva de concebir el conocimiento químico más allá de un robusto compendio de contenidos formales para considerarlo como un conjunto articulado de conocimientos específicos que exceden la dimensión disciplinar, demandando perspectivas de orden contextual filosófico e histórico que posibiliten el desplazamiento de la Educación Química de la simple contextualización a la problematización multifacética (SJÖSTRÖM; TALANQUER, 2014), en un proceso mediado por el gesto de interpretación en el que las formulaciones discursivas relativas a la Química se constituyan vestigio que permita observar no solamente las características propias de esta ciencia en sus niveles estructurantes - macroscópico, submicroscópico o simbólico (JOHNSTONE, 1982) -, sino que, como lo propone Sjöström (2013), se conceda un lugar preponderante al aspecto humanístico de la enseñanza de esta ciencia en al menos tres niveles de complejidad: Química Aplicada, SocioQuímica, y Química Crítica-Reflexiva (ilustración 1), lo cual, en conjunto, proporcione mayores elementos para comprender los modos de producción discursiva que posibilitan su materialización de sentidos, mediante diversas formas de textualización.

${ }^{3}$ Este término, entendido en la perspectiva pechêuxtiana del AD como el movimiento por el cual aprehendemos el proceso de producción de los sentidos y de los sujetos. 
Ilustración 1 - Tetraedro de Sjöström incluyendo diferentes niveles de complejidad en el análisis y discusión del elemento humano en la enseñanza de la Química

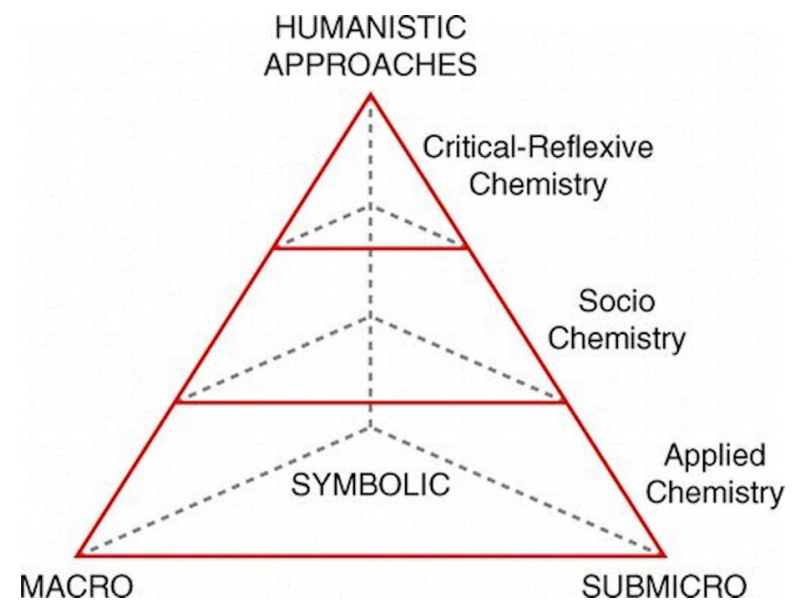

Química Crítico-Reflexiva: llama la atención de los estudiantes en el análisis reflexivo de las perspectivas históricas, filosóficas, sociológicas y culturales, así como también en la acción críticodemocrática por la socioecojusticia.

SocioQuímica: incluye aproximaciones apuntadas a la evaluación del desarrollo y usos del conocimiento químico, prácticas y productos, así como también la comprensión de inserciones socioculturales del trabajo y las ideas científicas.

Química Aplicada: caracteriza aproximaciones a la Educación Química que introducen el elemento humano enfocándose en asuntos de la vida diaria y diferentes aplicaciones de la Química.

Fuente: Sjöström y Talanquer (2014, p. 1126-1127, traducción nuestra).

Respecto a las formas de textualización, el DDErQ se puede materializar en el universo actual de posibilidades que ofrece lo digital de modos diferentes, por ejemplo,

- escrito (como textos posteados compuestos solamente por letras, números, signos de puntuación y caracteres);

- oral (como podcast y mensajes de voz);

- gráfico (como diseños o fotografías), también por la combinación de ellos como en el caso de las formas de comunicación paralingüísticas (tipo emoticones y emojis);

- gráfico-textual (tipo infografías o memes);

- gráfico-visual (tipo gifs y animaciones);

- audiovisual (tipo videoclips, documentales o series);

- multimedia (tipo simulaciones), o por las recientes formas de indexación mediante palabras clave, conocidas como Hashtag.

En este sentido, introducir la idea de que "[...] un texto producido en computador y un texto producido a mano son distintos en su orden porque las memorias que los moldean son distintas en sus materialidades: una es histórica y la otra es formal" (ORLANDI, 1996, p. 15, traducción nuestra) es fundamental en el propósito de configurar la noción de DDErQ, porque más allá de la técnica con que se da 'cuerpo' a la forma material de textualización, esa proposición remite a la necesidad de considerar en el mismo orden de importancia las condiciones de producción del discurso y la memoria.

Unas condiciones de producción que, de acuerdo con Orlandi (2003, p. 30, traducción nuestra), "[...] comprenden fundamentalmente los sujetos y la situación" y que, en un sentido amplio, "[...] incluyen el contexto socio-histórico, ideológico" en que cierta formulación discursiva se constituye, formula y circula, lo cual, en el AD se evidencia en que una forma material no significa por sí sola, aislada de sus condiciones de producción, porque "[...] las palabras no significan en sí. Ellas significan porque tienen textualidad, o sea, porque su interpretación deriva de un discurso que las sustenta, que las provee de realidad significativa" (ORLANDI, 2008b, p. 86, traducción nuestra). 
$Y$, una memoria que puede ser tratada como interdiscurso, es decir, como "[...] aquello que se habla antes, en otro lugar, independientemente. O sea, [...] lo que Ilamamos memoria discursiva" (ORLANDI, 2003, p. 31, traducción nuestra), una memoria que es constituida por el olvido y que 'reclama' sentido, lo que permite su historicización. Distinta de la memoria de archivo, que es institucional (ORLANDI, 2006a), de la memoria metálica, que es la memoria de la circulación, que funciona algorítmicamente, que se actualiza repetidamente y que no se historiciza (ORLANDI, 2006b), y de la memoria digital que, según Dias (2018, p. 105, traducción nuestra), "[...] seria [...], el lugar de la contradicción, donde la memoria escapa a la estructura totalizante de la máquina (memoria metálica), saliendo del espacio de la repetición formal y se inscribe en el funcionamiento del interdiscurso (memoria discursiva)".

A propósito de estos dos últimos conceptos una anotación resulta indispensable,

La memoria digital difiere de la memoria metálica, pero no se aparta de ella, pues si, por un lado, la memoria metálica funciona por la cantidad, por la posibilidad de almacenamiento y procesamiento de los datos, por otro lado, la memoria digital es un residuo que escapa a la estructura totalizante de la máquina y se inscribe en el funcionamiento del discurso digital, por el trabajo del interdiscurso. Por tanto, la memoria digital no es una simple re-actualización técnica de la memoria, o sea, una expansión horizontal de los enunciados, sino aquello que escapa a esa re-actualización, instalándose en la memoria discursiva, a través del trabajo del interdiscurso, considerando el acontecimiento de lo digital. (DIAS, 2018, p.161, traducción nuestra).

A manera de síntesis, los elementos teóricos pechêuxtianos que configuran las condiciones de producción de la noción de DDErQ refieren a las formaciones imaginarias, al interdiscurso, a la memoria digital y a la exterioridad en el campo de la enseñanza de la Química.

El cuarto y último elemento teórico de esta configuración surge de desplazar los procesos de enseñanza y de aprendizaje de la idea de la transmisión de conocimientos, para pensarlos en la idea de la transferencia de sentidos producidos (ORLANDI, 2001), proceso en el que la metaforización ocupa un lugar angular, pues, como lo refiere Dias (2018, p. 137, traducción nuestra):

La relación tecnología y enseñanza solo es posible si consideramos los sentidos de la metaforización de lo digital en el proceso educacional, lo que desplaza el sentido de una necesidad de adecuación de lo digital a lo educacional o de lo educacional a lo digital, para la historicización de los sentidos y de los sujetos, construcción de la historia de los sentidos, en la cual hay desplazamiento tanto de lo digital como del proceso educacional, en la medida en que trabajan en el intermedio de los dos discursos.

Esa articulación tecnología-enseñanza coloca en funcionamiento la necesidad de explorar recursos didácticos alternativos, como los de naturaleza digital, considerando que las actuales condiciones de producción de enseñar y de aprender están atravesadas por la generación de conocimiento en red, que circula en forma de grandes cantidades de información, que corresponden a recortes de memoria en un punto del interdiscurso, que se textualizan en el intradiscurso, y se estabilizan por la repetición.

En ese encadenamiento, abandonar el eje de lo repetible, del dato, romper el funcionamiento algoritmizado para historicizar recortes del conocimiento químico 
que circulan por lo digital, desplazándose a ese lugar de contradicción que Dias (2018) llama memoria digital, el papel del recurso didáctico es importante y no meramente instrumental en la producción del DDErQ. Sin embargo, tal como lo señala Almeida (2012, p. 38, traducción nuestra), es crucial no olvidar que,

[...] cualquier recurso es apenas un recurso, con determinados potenciales. Su uso depende de como es organizada el aula por los profesores formadores de formadores o por los profesores de enseñanza básica. Estos, más allá de direccionar la naturaleza de las actividades propuestas con el uso de los recursos disponibles, son los mediadores de la circulación de los discursos en el salón de clase.

En consecuencia, y tal como se ha planteado recurrentemente, no se trata de abordar lo digital como una cuestión secundaria o accesoria, pues, sin duda, en el mundo hiperconectado en el que vivimos, "[...] la relación hombre-máquina es una relación que ya hace parte de la propia constitución de los sujetos" (DIAS, 2018, p. 49, traducción nuestra), que pasan de ser considerados 'usuarios' a ser reconocidos como 'sujetos de datos', conforme coloca la autora, lo cual remite a la figura de un sujeto interpelado, individualizado, capital fundamental de la propia construcción de las plataformas tecnológicas, fuente de informaciones que son algoritmizadas por la máquina, y que facilitan su caracterización en el universo digital. Y que, no menos importante, se encuentra inmerso en un proceso de inscripción en la sociedad; escenario en el que la escuela adquiere un papel determinante en función de: (1) ofrecerle herramientas para asumir una posición-sujeto en el interior de formaciones discursivas específicas; (2) reconocer y establecer comunicaciones dinámicas con su entorno, entre otras, atravesadas por los datos que disponibiliza al sistema y los que circulan disponibilizados por otros sujetos; y, (3) relacionarse activamente con los distintos efectos de sentido que pueden ser producidos alrededor de un asunto, en este caso puntual, respecto al conocimiento químico.

En esa coyuntura, conviene repensar otras formas de autoría en lo digital, como aquellas observadas por Orlandi (2008b, p. 183, traducción nuestra):

[...] en relación con lo público, a la multiplicación de la repetición y a la cantidad. Eso ciertamente hace efecto sobre la forma función-autor y, correspondientemente, del efecto-lector. Si la memoria metálica se organiza en nuevos modos de (no) olvido, si la metáfora se da en otra instancia, también la textualización procede diferentemente y carga consigo otras formas de autoría.

De este modo, reconociendo que, en la materialización del discurso, otras cosas fueron dichas con antecedencia y otras serán dichas con posterioridad al respecto de cierto asunto, en la noción de DDErQ el profesor en formación inicial se coloca en la fuente del decir para producir un efecto imaginario de unidad de sentido, configurando una textualidad.

En este escenario, considerar al profesor en formación inicial como autor implica dejar de pensarlo como propietario de un decir, para reconocerlo en los términos de Orlandi (2008a, p. 77, traducción nuestra), como la "[...] función que el yo asume en cuanto productor de lenguaje". En otras palabras, en la dimensión de sujeto creador de un lugar de interpretación, inscrito en las reglas de la institucionalidad escolar, que a su vez, se inscriben en medio de otros lugares de interpretación, cuyas reglas están determinadas por la relación con la exterioridad (contexto socio-histórico). 
Por supuesto, no es posible dejar de referir que el proceso de significación es histórico y no técnico, por lo que, concordando con Orlandi (2008b, p. 64, traducción nuestra) "[...] explicitar los mecanismos de producción de sentidos inscritos en el texto es una manera de tornar visible el modo como la exterioridad (sujeto, historia) está presente en él, es trabajar en su historicidad", lo que puede producir un efecto favorable sobre las prácticas de formación inicial del profesorado, en la medida en que se configuren nuevos espacios para comprender, como apunta Almeida (2010, p. 20, traducción nuestra), que "[...] diferentes discursos relativos a la ciencia [en este caso, relativos a la Química] en circulación en la escuela van a ser interpretados según las condiciones de producción inmediatas de enseñanza, pero también según las diferentes historias de vida de los que frecuentan aquella institución". Ese posicionamiento concede al futuro profesor un lugar interpretativo, y no meramente repetitivo, en la construcción colectiva del conocimiento químico escolar.

Es así que, ampliando los límites geométricos estáticos de la propuesta tetraédrica de Sjöström (2013), en su funcionamiento conjunto, los cuatro pilares teóricos del DDErQ resumen un complejo sistema de engranajes que solidariamente apunta a la comprensión del orden del discurso digital escolar en función de producir efectos de sentido en las prácticas de formación inicial del profesorado, que (se espera) contribuyan a la contextualización, a la historización, y a la humanización en la futura enseñanza de la Química.

\section{EI DDErQ y sus posibles implicaciones en el campo de la enseñanza de la Química}

Previo a este apartado final, conviene destacar que la proposición de esta noción hace parte de una tesis doctoral en desarrollo, en consecuencia, los casos concretos de estudio y su aplicación se publicarán posteriormente.

En virtud de lo expuesto, la noción de DDErQ tejida en la filiación pechêuxtiana del $A D$, teóricamente representa una posibilidad para reflexionar y transformar las futuras prácticas escolares de la Enseñanza de la Química, considerando desde la formación inicial del profesorado:

1. Una enseñanza de la Química que abandona el contenido enciclopédico y la concepción ingenua, mecánica y reduccionista de esta ciencia, como simple replicadora de conceptos y procedimientos que constituyen procesos y productos útiles para la sociedad, y que 'deben ser' conocidos por los ciudadanos para promover tomas de decisiones democráticas más consientes e informadas. Por consiguiente, pensar la enseñanza de la Química desde el AD supone que no es posible ignorar la historicidad de los conceptos que configuran el conocimiento químico, la inmensa red de conexiones que se pueden establecer entre ellos, sus especificidades, pero también sus límites; desconocer sus particularidades en tanto ciencia objeto de enseñanza, o pretender que su enseñanza y su aprendizaje están aislados de la subjetividad, de la falla o de la incompletitud del lenguaje.

2. Una configuración de modos de producción discursiva propios de la enseñanza de la Química en una sociedad digital, basados en una nueva relación entre la Química, la Educación, la Tecnología y las prácticas discursivas, que pasa por el reconocimiento de las características particulares de las formaciones discursivas relativas a esta ciencia en sus niveles estructurantes, transversalizadas por el 
carácter humanístico en los procesos de formación inicial del profesorado, en donde resulta imperativo concebir a los futuros educadores como sujetos de datos, y a los medios materiales de comunicación (tipo computadores personales, smartphones y tablets) como potenciales mediadores en la producción discursiva de naturaleza científico-escolar.

3. Una narratividad que se produce en desarrollo de las actividades escolares en espacios de escritura, como el de los medios digitales (DIAS, 2018) que pasan de pensarse como simples apoyos tecnológicos que facilitan el proceso de enseñanza y de aprendizaje, a funcionar como robustos recursos didácticos en la mediación del DDErQ, producto de una relectura de lo digital, en la que los objetos y los sujetos se resignifican.

4. Un escenario fructífero para la construcción de unidad en medio de la dispersión y de la polisemia, que favorezca la transformación de las futuras prácticas escolares de la enseñanza de la Química, pensando otras maneras de abordar las cuestiones relacionadas con el acceso de los estudiantes a los diferentes conocimientos que hacen parte de su andamiaje conceptual, pero también, apuntando hacia la comprensión de sus aplicaciones, el análisis crítico de sus desafíos actuales y de sus disputas históricas, y la discusión respecto de su papel activo en la toma de decisiones, tanto individuales, como colectivas, informadas, responsables, éticas y democráticas.

\section{Agradecimientos}

A la Coordenação de Aperfeiçoamento de Pessoal de Nível Superior (CAPES) por el apoyo económico brindado para el desarrollo de esta investigación a través del Programa Estudiante - Convenio de Posgraduación (PEC-PG) y al Profesor Doctor Pedro da Cunha Pinto Neto por sus valiosas contribuciones en calidad de orientador de la tesis en desarrollo.

\section{Referencias}

ALMEIDA, M. J. P. M. Discurso pedagógico e formação de professores das ciências da natureza: foco no professor de física. Alexandria, Florianópolis, v. 5, n. 2, p. 29-41, 2012. Recuperado el 9 mar. 2020 de: https://cutt.ly/7QqvpnP.

ALMEIDA, M. J. P. M. O texto de divulgação cientifica como recurso didático na mediação do discurso escolar relativo à ciência. In: PINTO, G. (org.). Divulgação cientifica e práticas educativas. Curitiba: CRV, 2010. p. 11-24.

CARVALHO, A. M. P.; GIL-PÉREZ, D. Formação de professores de ciências: tendências e inovações. 10. ed. São Paulo: Cortez, 2011.

CHANG, R.; GOLDSBY, K. Química. 11. ed. México: McGraw Hill Education, 2013.

DIAS, C. Análise do discurso digital: sujeito, espaço, memória e arquivo. Campinas: Pontes, 2018.

DIAS, C. Textualidades seriadas: entre a repetição, a regularização e o deslocamento, o caso dos memes. RASAL Lingüística, Buenos Aires, n. 2, p. 54-74, 2019.

JOHNSTONE, A. Macro and microchemistry. School Science Review, London, v. 64, n. 227, p. 377$379,1982$. 
LABARCA, M. Acerca de la naturaleza de la química: algunos comentarios. Educación en la Química, Buenos Aires, v. 15, n. 2, p. 89-102, 2009. Recuperado el 24 jul. 2021 de: https://cutt. ly/0QqvHOJ.

ORLANDI, E. Análise de discurso: introdução. In: ORLANDI, E.; LAGAZZI-RODRIGUES, S. (org.). Introdução às ciências da linguagem: discurso e textualidade. Campinas: Pontes, 2006a. p. 11-28.

ORLANDI, E. Análise de discurso: princípios e procedimentos. Campinas: Pontes, 2003.

ORLANDI, E. Discurso e leitura. 8. ed. São Paulo: Cortez, 2008a.

ORLANDI, E. Discurso e texto: formulação e circulação de sentidos. 3. ed. Campinas: Pontes, 2008b.

ORLANDI, E. História das ideias x história de vida: entrevista com Eni Orlandi. [Entrevista cedida a] Amanda Scherer. Fragmentum, Santa Maria, n. 7, p. 11-51, 2006b. Recuperado el 26 jul. 2021 de: https://cutt.ly/MQrnxYw.

ORLANDI, E. Interpretação: autoria, leitura e efeitos do trabalho simbólico. Petrópolis: Vozes, 1996.

ORLANDI, E. Para quem é o discurso pedagógico? In: ORLANDI, E. A linguagem e seu funcionamento: as formas do discurso. 4. ed. Campinas: Pontes, 2001. p. 18-31.

ORLANDI, E. Tecnologias da linguagem: um novo funcionamento. In: ORLANDI, E. O que é linguística? 2. ed. São Paulo: Brasiliense, 2009.

PÊCHEUX, M. Analyse automathique du discours. Paris: Dunod, 1969.

PÊCHEUX, M. Semântica e discurso: uma crítica à afirmação do óbvio. Campinas: Ed. Unicamp, 1988.

SJÖSTRÖM, J. Towards bildung-oriented chemistry education. Science \& Education, Dordrecht, v. 22, n. 7, p. 1873-1890, 2013. DOI: https://doi.org/bfn2cv.

SJÖSTRÖM, J., TALANQUER, V. Humanizing chemistry education: from simple contextualization to multifaceted problematization. Journal of Chemistry Education, Washington, v. 91, n. 8, p. 11251131, 2014. DOI: https://doi.org/10.1021/ed5000718.

TABER, K. S. Revisiting the chemistry triplet: drawing upon the nature of chemical knowledge and the psychology of learning to inform chemistry education. Chemistry Education Research and Practice, London, v. 14, n. 2, p. 156-168, 2013. DOI: https://doi.org/gdpff2.

TALANQUER V. Macro, submicro, and symbolic: the many faces of the chemistry "triplet". International Journal of Science Education, London, v. 33, n. 2, p. 179-195, 2011. DOI: https://doi. org/dsnfbf.

TALANQUER, V. Formación docente: ¿qué conocimientos distingue a los buenos maestros de química? Educación Química, Ciudad de México, v. 15, n. 1, p. 61-66, 2004. DOI: https://doi.org/ gpxb. 